\title{
Editorial \\ NIELSEN THEORY AND RELATED TOPICS
}

\author{
PHILIP R. HEATH, ROBERT F. BROWN, AND EDWARD C. KEPPELMANN
}

Received 14 July 2005; Accepted 14 July 2005

Copyright (C 2006 Philip R. Heath et al. This is an open access article distributed under the Creative Commons Attribution License, which permits unrestricted use, distribution, and reproduction in any medium, provided the original work is properly cited.

The International Conference on Nielsen Theory and Related Topics took place from June 28 through July 2, 2004 at Memorial University, St. John's, Newfoundland, Canada. This was the 13th such conference in a series that began in 1977 with a conference in Oberwolfach, Germany.

Nielsen theory is named after Jakob Nielsen who, in the 1920s, turned the focus of fixed point theory from the existence of fixed points (as, e.g., in the famous Lefschetz fixed point theorem) to the problem of estimating the actual number of such points within the homotopy class of a given map. He did this by introducing what is now called the Nielsen number of a self map, a homotopy invariant lower bound for the number of fixed points of the map. After important initial contributions by Reidemeister and Wecken, there was little activity in Nielsen theory until the 1960s when a breakthrough by Boju Jiang allowed for easy calculations of the Nielsen number for maps on Lie groups and some other interesting kinds of spaces. It was these and other important examples that would guide the direction of research. As the present collection of papers illustrates, the frontiers of the subject now involve an impressive variety and interplay of algebraic and geometric techniques, on a wide class of spaces. Consequently, there continue to be an increasing number of interesting areas for future investigations, both in the areas of computation and the development of new invariants.

As research in Nielsen theory progressed, its concern with fixed points expanded, on the one hand, into related issues such as coincidences, periodic points, and roots and, on the other, into various refinements for restricted classes of maps and homotopies such as those that occur in the fiber space, relative and equivariant contexts. Just about all of these aspects of Nielsen theory were represented by the talks in Newfoundland. The conference 
was attended by 36 people from 14 different countries and five of the six inhabitable continents; this comprises almost all mathematicians who are currently active in research in Nielsen theory. The participants displayed a range of experience ranging from graduate students to people who had attended the previous Canadian Nielsen theory conference, in Sherbrooke, Quebec, in 1980. The group also included a number of experienced mathematical researchers who are new to Nielsen theory. Their varied expertise is providing important new directions for research in our subject.

The major financial support for the conference was furnished by Canada's Atlantic Association for Research in the Mathematical Sciences (AARMS) which, in turn, is partially funded by Memorial University of Newfoundland (MUN). We are profoundly grateful for the generous support from AARMS. Substantial funding for the attendance of participants from developing countries came from the Commission on Development and Exchanges (CDE) of the International Mathematical Union (IMU). We thank Prof. Herbert Clemens, Secretary of the CDE, and also the Executive Committee of the IMU which modified its rules in order to support these mathematicians at a conference not held in a developing country. Special thanks are due to the secretaries of the MUN Mathematics Department, in particular to Wanda Heath and Ros English, for their outstanding help in organizing the conference.

We are also grateful to Prof. Ravi Agarwal and the publishers of the journal "Fixed Point Theory and Applications" for the opportunity to celebrate the occasion of the Newfoundland conference by publishing the collection of research papers in Nielsen theory that occupy this issue.

Philip R. Heath

Robert F. Brown

Edward C. Keppelmann 